\title{
Combined evaluation of coronary artery disease and high-sensitivity cardiac troponin $T$ for prediction of adverse events inpatients with hypertrophic cardiomyopathy
}

\author{
Hang Liao ${ }^{1}$, Huay Cheem Tan, Ziqiong Wang ${ }^{1}$, Xiaoping Chen ${ }^{1}$, Yong $\mathrm{He}^{1}$ and Sen $\mathrm{He}^{1 *}$ (i)
}

\begin{abstract}
Background: This study was performed to investigate the clinical significance of combined evaluation of both coronary artery disease (CAD) and high-sensitivity cardiac troponin T (hs-cTnT) for prediction of major adverse cardiovascular events (MACEs) in patients with hypertrophic cardiomyopathy (HCM).

Methods: We performed clinical evaluations, including coronary artery imaging and hs-cTnT measurement, in 162 patients with HCM.

Results: The patients were followed up for a median period of 3.7 years (interquartile range 2.4-5.6 years; total of 632.3 person-years [PYs]), during which time MACEs occurred in $24(14.8 \%)$ patients. The incidence of MACEs was 6.4 and 2.7 per $100 \mathrm{PYs}$ for patients with CAD and normal coronary arteries, respectively; similarly, the incidence was 5.8 and 2.1 per 100 PYs in patients with an elevated hs-CTnT concentration (>14.0 ng/L) and a normal hs-cTnT concentration, respectively. The multivariate analysis suggested that CAD and an elevated hs-cTnT concentration tended to be positively associated with MACEs. When the groups were allocated according to these two markers, the patients were divided into four groups, which further improved the predictive values. The incidence of MACEs was 10.4 per 100 PYs in the CAD and elevated hs-cTnT group, which was much higher than the incidence in all other groups (range, 2.0-3.5 per 100 PYs). With the normal coronary arteries and normal hs-cTnT group serving as a reference, the adjusted hazard ratio was 5.0 (95\% confidence interval 1.0-23.8; $P=0.046$ ) for the CAD and elevated hs-cTnT group. In addition, the subgroup analysis showed similar findings among the patients without severe CAD.
\end{abstract}

Conclusions: In patients with HCM, combined evaluation of both CAD and hs-CTnT might facilitate more reliable prediction of MACEs than evaluation of a single marker. These may serve as clinically useful markers to guide risk management.

Keywords: Hypertrophic cardiomyopathy, High-sensitivity cardiac troponin T, Coronary artery disease, Major adverse cardiovascular events

*Correspondence: xiaopingchen11@163.com

1 Department of Cardiology, West China Hospital of Sichuan University, Chengdu 610041, China

Full list of author information is available at the end of the article

\section{Background}

Hypertrophic cardiomyopathy (HCM) is the most commonly inherited cardiovascular disease, with a prevalence of $0.2 \%$ in the general population. It has a higher prevalence $(0.5 \%)$ when both clinical and genetic diagnoses are taken into account [1-3]. HCM has been regarded 
as having a poor prognosis with limited management options. During the last 2 decades, important advances in contemporary management strategies have greatly improved the life expectancy and quality of patients with HCM [3-5].

However, some recent studies have suggested that other cardiac or non-cardiac comorbidities might have a greater impact on survival than long-standing HCM itself $[6,7]$. For example, some studies have shown that coronary artery disease (CAD) is associated with an increase in poor prognosis in patients with $\operatorname{HCM}[8,9]$. The serum concentration of high-sensitivity cardiac troponin $\mathrm{T}$ (hs-cTnT), a sensitive and specific marker of myocardial injury, is also reportedly effective in predicting adverse outcomes in patients with $\operatorname{HCM}[10,11]$. In general, CAD can cause a decrease in coronary blood flow to different degrees, and in patients who have HCM without myocardial infarction, an elevated hs-cTnT concentration may indicate microvascular dysfunction $[10$, 12]. These parameters might represent the total ischemic burden of the myocardium in HCM, which is considered to be associated with poor outcomes. However, whether evaluations of CAD and hs-cTnT might supplement each other and thus become more reliable prognostic markers in patients with HCM remains unclear. The present study was therefore performed to examine the efficiency of combined evaluation of these two markers for prediction of adverse events in patients with HCM.

\section{Methods}

\section{Study population}

This retrospective, single-center, longitudinal study was performed at West China Hospital of Sichuan University (a tertiary referral center in Chengdu, China). From December 2008 to May 2016, 508 consecutive inpatients with a diagnosis of HCM were enrolled in the study. The diagnosis of HCM was based on a wall thickness of $\geq 15 \mathrm{~mm}$ in one or more left ventricular (LV) myocardial segments as measured by transthoracic echocardiography or cardiac magnetic resonance imaging that could not be solely explained by abnormal loading conditions [1]. The patients' data were input twice by medical professionals. If any inconsistency was encountered, the data were rechecked. Patients who were diagnosed with inherited metabolic diseases or syndromic causes of $\mathrm{HCM}$ were excluded from the study. Other detailed information were reported in a recently published study [13]. The final study population comprised 162 patients (Fig. 1), none of whom had a history of coronary stent implantation before the first evaluation.

The study was approved by the Ethics Committee on Medical Research of West China Hospital of Sichuan University and conformed to the principles of the
Helsinki declaration. The requirement for informed consent was waived because of the retrospective nature of the study.

\section{Clinical evaluation}

Evaluation of patients included a medical history, clinical examination, and Doppler echocardiography. All patients underwent a two-dimensional transthoracic echocardiography examination conducted by a single experienced echocardiographer. LV hypertrophy, LV end-diastolic dimension, LV ejection fraction, LV outflow tract (LVOT) pressure gradient, and left atrial (LA) diameter were assessed by standard techniques. Maximum LV wall thickness was defined as the greatest thickness in any single segment, and LVOT obstruction was defined as a peak LVOT gradient of $\geq 30 \mathrm{mmHg}$ in the resting state $[1,9]$.

\section{Evaluation of CAD and hs-cTnT}

The severity of CAD was assessed by coronary angiography $(n=129)$ or coronary computed tomography (CT) angiography $(n=33)$. Severe CAD was defined as a single luminal diameter stenosis of $\geq 50 \%$ in the left main coronary artery or $\geq 70 \%$ in other major epicardial branches or the presence of two luminal diameter stenoses of $\geq 50 \%$. Mild to moderate CAD was defined as luminal diameter stenoses that did not meet the criteria for severe CAD [8]. In addition, patients who agreed to undergo percutaneous coronary intervention $(\mathrm{PCI})$ at the baseline evaluation were also defined as having severe CAD.

CAD was severe in 10 patients, and 5 agreed to undergo PCI at baseline. CAD was mild to moderate in 45 patients, and none of these patients agreed to undergo PCI. Because of the small number of patients with severe CAD, we combined these patients with those who had mild to moderate CAD to form a new group (CAD group, $\mathrm{n}=55$ ). A total of 107 patients had normal coronary arteries (NCA); among them, 0 had left main disease $(\geq 50 \%)$, 8 had proximal lesions of the left anterior descending branch ( $\geq 50 \%)$, 2 had right coronary artery disease ( $\geq 50 \%)$, and 3 had left circumflex artery disease $(\geq 50 \%) .5$ in one vessels with luminal stenosis $\geq 50 \%, 4$ in two vessels with luminal stenosis $\geq 50 \%$, and 1 in three vessels with luminal stenosis $\geq 50 \%$. The hs-cTnT concentration was assayed using electrochemiluminescence according to the manufacturer's instructions (Roche Diagnostics, Mannheim, Germany). The reference range of hs-cTnT in an apparently healthy adult population is $\leq 14 \mathrm{ng} / \mathrm{L}$ (99th percentile) [14]. Seventy-seven patients had an hs-cTnT concentration within the reference range, and 85 had an elevated hs-cTnT concentration. 


\section{Patients diagnosed as having hypertrophic} cardiomyopathy in Dec 2008-May 2016

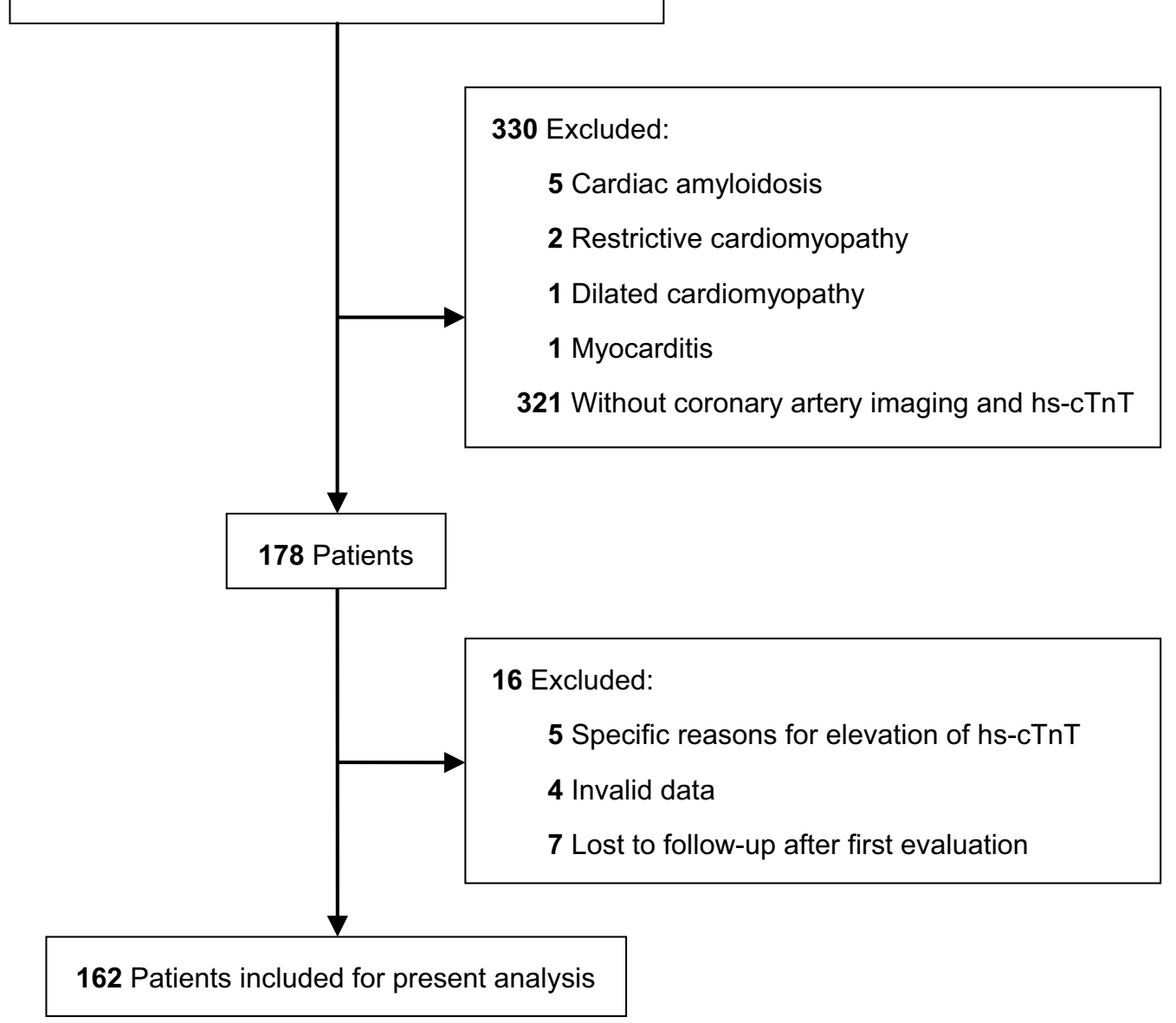

Fig. 1 Study flow diagram

\section{Follow-up evaluation}

Follow-up was carried out via medical records and telephone contact with patients themselves or their referring relatives. All patients were followed from the first evaluation to the endpoint or the most recent evaluation.

The primary endpoint of this study was any major adverse cardiovascular events (MACEs), defined as a composite of cardiovascular death, a thromboembolic event, and myocardial infarction/PCI. Cardiovascular death was defined as mortality resulting from any cardiovascular disease, including sudden cardiac death, heart failure-related death, myocardial infarction-related death, perioperative cardiac death, or appropriate shock from an implantable cardioverter defibrillator that was equal to sudden cardiac death. Thromboembolic events included ischemic stroke, transient ischemic attack, and peripheral embolism.

\section{Statistical analysis}

Descriptive statistics (mean \pm standard deviation, percentage, etc.) were used to summarize the baseline characteristics. Differences in continuous variables were assessed using one-way analysis of variance, and categorical variables were compared by the chi-square test. The patients were divided into four new groups to assess the associations of the two markers with the primary endpoint: those with NCA and a normal hs-cTnT concentration, those with CAD and a normal hs-cTnT concentration, those with NCA and an elevated hs-cTnT concentration, and those with CAD and an elevated hscTnT concentration. MACEs were graphically represented using Kaplan-Meier curves, and the log rank test 
was used for comparison. Baseline variables that were considered clinically relevant or that showed a univariate relationship with the outcome $(P<0.1)$ were entered into a multivariate Cox proportional hazards regression model. Variables for inclusion were carefully chosen, given the number of events available, to ensure parsimony of the final model. Additionally, we fitted a multivariable model as a sensitivity analysis using Lasso regression, a penalized regression method suitable for data sets with few events [15]. We used Lasso regression to select the potential variables on the basis of lambda. min and constructed a multivariate Cox model. Moreover, we reported the net reclassification index with hscTnT in addition to the presence of CAD.

All analyses were performed with $\mathrm{R}$ version 3.6.3, including the "compareGroups," "rms," "survminer," "tidyverse," "glmnet," "nricens," and "base" packages (http://www.R-project.org). All tests were two-sided, and $P$ values of $<0.05$ were considered statistically significant.

\section{Results}

\section{Baseline characteristics}

Compared with the patients in the NCA group, the patients in the CAD group were older and had higher incidences of hypertension, diabetes, current or prior tobacco use, vascular diseases, and LVOT obstruction. In addition, the CAD group had more prescriptions of aspirin, clopidogrel, and statins. The patients in the elevated hs-cTnT group were older and more commonly had a history of vascular disease and New York Heart Association (NYHA) class III/IV heart disease than the patients in the normal hs-cTnT group. The patients in the elevated hs-cTnT group had a larger LA diameter and maximum LV wall thickness, a higher high-density lipoprotein cholesterol concentration, and a lower LV ejection fraction (detailed data not shown).

According to the four newly defined groups mentioned above, the NCA and normal hs-cTnT group comprised 54 patients, the CAD and normal hs-cTnT group comprised 23 patients, the NCA and elevated hs-cTnT group comprised 53 patients, and the CAD and elevated hs-cTnT group comprised 32 patients. The differences among the four groups are presented in Table 1.

\section{Follow-up data}

The patients were followed for a median period of 3.7 years (interquartile range $2.4-5.6$ years; total, 632.3 person-years [PYs]), and 24 (14.8\%) reached the primary endpoint. Clearly defined coronary-related mortality and morbidity developed in about $20.8 \%$ of events (Table 2 ). MACEs more frequently occurred in the CAD group than NCA group (log-rank $P=0.031$ ) (Fig. 2A; specifically, $12(21.8 \%)$ MACEs occurred in the CAD group and 12 (11.2\%) MACEs occurred in the NCA group. The incidence of MACEs per 100 PYs was 6.4 (95\% confidence interval [CI] 2.9-10.0) and 2.7 (95\% CI 1.2-4.2) for patients with CAD and NCA, respectively.

The elevated hs-cTnT group had more MACEs than the normal hs-cTnT group (log-rank $P=0.015$ ) (Fig. 2B). Specifically, MACEs occurred in 17 (20.0\%) patients in the elevated hs-cTnT group and in 7 (9.1\%) patients in the normal hs-cTnT group. The incidence of MACEs was 5.8 (95\% CI 3.1-8.4) and 2.1 (95\% CI 0.6-3.6) per 100 PYs in the elevated hs-cTnT group and normal hs-cTnT group, respectively.

Among the newly defined groups, the Kaplan-Meier curve showed that the clinical course was significantly worse in the CAD and elevated hs-cTnT group (log-rank $P=0.003$ ) (Fig. 2C); there was no difference among the other three groups (log-rank $P=0.582$ ) (Fig. 2 C). Table 2 shows the specific MACEs in the different groups. MACEs occurred in $5(9.3 \%)$ patients in the NCA and normal hs-cTnT group, 2 (8.7\%) patients in the CAD and normal hs-cTnT group, 7 (13.2\%) patients in the NCA and elevated hs-cTnT group, and $10(31.3 \%)$ patients in the CAD and elevated hs-cTnT group. The incidence of MACEs was 10.4 (95\% CI 4.3-16.6) per 100 PYs in the CAD and elevated hs-cTnT group, which was much higher than that in the other three groups (Table 3).

\section{Univariate and multivariate analyses}

The univariate analysis showed seven variables with a $P$ value of $<0.1$ : age, CAD, hs-cTnT, combined evaluation, LA diameter, the use of statins, and the use of beta-blockers.

The multivariate analysis showed that CAD and hscTnT tended to be positively associated with MACEs. With the NCA or normal hs-cTnT group as a reference, the adjusted hazard ratios (HRs) were 1.7 (95\% CI 0.55.6; $P=0.371)$ and $2.8(95 \%$ CI $1.0-8.1 ; P=0.056)$ for the CAD or elevated hs-cTnT group, respectively.

The multivariate analysis indicated that combined evaluation of both CAD and hs-cTnT was positively associated with MACEs, which further improved the prognostic values. With the NCA and normal hs-cTnT group as reference, the adjusted HRs were 0.5 (95\% CI 0.1-3.9; $P=0.542)$ for the CAD and normal hs-cTnT group, 1.2 (95\% CI 0.3-5.2; $P=0.765$ ) for the NCA and elevated hs-cTnT group, and 5.0 (95\% CI 1.0-23.8; $P=0.046)$ for the CAD and elevated hs-cTnT group (Table 3). Moreover, the multivariate analysis also suggested that the LA diameter (per 1-mm increase; HR 1.066; 95\% CI 0.9971.139; $P=0.054$ ), age (per 1-year increase; HR 1.050; $95 \%$ CI 0.996-1.106; $P=0.069$ ), and use of aspirin (HR 0.273; 95\% CI $0.069-1.081 ; P=0.068)$ tended to be predictors of MACEs. However, these results should be interpreted 
Table 1 Baseline characteristics

\begin{tabular}{|c|c|c|c|c|c|c|}
\hline Variables & All patients $(n=162)$ & $\begin{array}{l}\text { NCA, normal } \\
\text { hs-cTnT }(n=54)\end{array}$ & $\begin{array}{l}\text { CAD, normal } \\
\text { hs-cTnT }(n=23)\end{array}$ & $\begin{array}{l}\text { NCA, elevated } \\
\text { hs-cTnT }(n=53)\end{array}$ & $\begin{array}{l}\text { CAD, elevated } \\
\text { hs-cTnT }(n=32)\end{array}$ & $P$ value \\
\hline Age (years) & $57.5 \pm 13.5$ & $52.2 \pm 12.5$ & $63.3 \pm 11.5$ & $55.9 \pm 14.4$ & $64.6 \pm 10.1$ & $<0.001$ \\
\hline Gender, male & $95(58.6 \%)$ & $31(57.4 \%)$ & $16(69.6 \%)$ & $30(56.6 \%)$ & $18(56.2 \%)$ & 0.722 \\
\hline Family history of HCM & $10(6.2 \%)$ & $3(5.6 \%)$ & $1(4.3 \%)$ & $4(7.5 \%)$ & $2(6.2 \%)$ & 0.952 \\
\hline Family history of SCD & $4(2.5 \%)$ & $1(1.9 \%)$ & $1(4.3 \%)$ & $2(3.8 \%)$ & $0(0.0 \%)$ & 0.658 \\
\hline Current or prior tobacco use & $60(37.0 \%)$ & $18(33.3 \%)$ & $13(56.5 \%)$ & $15(28.3 \%)$ & $14(43.8 \%)$ & 0.093 \\
\hline NYHA class III/IV & $52(32.1 \%)$ & $10(18.5 \%)$ & $7(30.4 \%)$ & $22(41.5 \%)$ & $13(40.6 \%)$ & 0.050 \\
\hline \multicolumn{7}{|l|}{ Symptoms } \\
\hline Chest pain & $121(74.7 \%)$ & $41(75.9 \%)$ & $18(78.3 \%)$ & $35(66.0 \%)$ & $27(84.4 \%)$ & 0.274 \\
\hline Palpitations & $64(39.5 \%)$ & $22(40.7 \%)$ & $8(34.8 \%)$ & $23(43.4 \%)$ & $11(34.4 \%)$ & 0.816 \\
\hline Syncope/pre-syncope & $47(29.0 \%)$ & $16(29.6 \%)$ & $7(30.4 \%)$ & $20(37.7 \%)$ & $4(12.5 \%)$ & 0.101 \\
\hline Dyspnea & $98(60.5 \%)$ & $29(53.7 \%)$ & $13(56.5 \%)$ & $34(64.2 \%)$ & $22(68.8 \%)$ & 0.493 \\
\hline \multicolumn{7}{|l|}{ Comorbidities } \\
\hline Atrial fibrillation & $18(11.1 \%)$ & $4(7.4 \%)$ & $4(17.4 \%)$ & $5(9.4 \%)$ & $5(15.6 \%)$ & 0.479 \\
\hline Hypertension & 64 (39.5\%) & $14(25.9 \%)$ & 14 (60.9\%) & $15(28.3 \%)$ & $21(65.6 \%)$ & $<0.001$ \\
\hline Diabetes & 15 (9.3\%) & $4(7.4 \%)$ & $3(13.0 \%)$ & $3(5.7 \%)$ & $5(15.6 \%)$ & 0.396 \\
\hline Vascular diseases & $18(11.1 \%)$ & $1(1.9 \%)$ & $0(0.0 \%)$ & $5(9.4 \%)$ & $12(37.5 \%)$ & $<0.001$ \\
\hline \multicolumn{7}{|l|}{ Therapies } \\
\hline Aspirin & $38(23.5 \%)$ & $8(14.8 \%)$ & $8(34.8 \%)$ & $6(11.3 \%)$ & $16(50.0 \%)$ & $<0.001$ \\
\hline Clopidogrel & $14(8.6 \%)$ & $2(3.7 \%)$ & $1(4.3 \%)$ & $3(5.7 \%)$ & $8(25.0 \%)$ & 0.003 \\
\hline Warfarin & $8(4.9 \%)$ & $2(3.7 \%)$ & $0(0.0 \%)$ & $3(5.7 \%)$ & $3(9.4 \%)$ & 0.428 \\
\hline Statins & $69(42.6 \%)$ & $15(27.8 \%)$ & $15(65.2 \%)$ & $15(28.3 \%)$ & $24(75.0 \%)$ & $<0.001$ \\
\hline Beta-blocker & $131(80.9 \%)$ & $44(81.5 \%)$ & 21 (91.3\%) & 42 (79.2\%) & $24(75.0 \%)$ & 0.487 \\
\hline Diltiazem & $10(6.2 \%)$ & $4(7.4 \%)$ & $2(8.7 \%)$ & $2(3.8 \%)$ & $2(6.2 \%)$ & 0.820 \\
\hline$I C D$ & $7(4.3 \%)$ & $1(1.9 \%)$ & $0(0.0 \%)$ & $5(9.4 \%)$ & $1(3.1 \%)$ & 0.446 \\
\hline Pacemaker & $4(2.5 \%)$ & $1(1.9 \%)$ & $1(4.3 \%)$ & $1(1.9 \%)$ & $1(3.1 \%)$ & \\
\hline Septal myectomy & $1(0.6 \%)$ & $0(0.0 \%)$ & $0(0.0 \%)$ & $1(1.9 \%)$ & $0(0.0 \%)$ & 0.293 \\
\hline Alcohol septal ablation & $28(17.3 \%)$ & $14(25.9 \%)$ & $2(8.7 \%)$ & $6(11.3 \%)$ & $6(18.8 \%)$ & \\
\hline \multicolumn{7}{|l|}{ Echocardiography data } \\
\hline $\operatorname{LVEDD~(mm)~}$ & $44.4 \pm 5.6$ & $43.9 \pm 5.1$ & $44.7 \pm 4.8$ & $44.6 \pm 6.9$ & $44.7 \pm 4.5$ & 0.883 \\
\hline LA diameter (mm) & $40.7 \pm 6.8$ & $38.8 \pm 6.3$ & $40.4 \pm 7.2$ & $42.3 \pm 7.3$ & $41.3 \pm 5.9$ & 0.056 \\
\hline MWT (mm) & $18.9 \pm 4.8$ & $18.6 \pm 5.0$ & $17.1 \pm 3.1$ & $20.0 \pm 5.4$ & $19.1 \pm 4.1$ & 0.108 \\
\hline LVEF (\%) & $67.5 \pm 8.6$ & $70.3 \pm 4.7$ & $68.9 \pm 5.2$ & $65.3 \pm 11.9$ & $65.6 \pm 7.8$ & 0.008 \\
\hline LVOT obstruction & $78(48.1 \%)$ & $27(50.0 \%)$ & $11(47.8 \%)$ & $17(32.1 \%)$ & $23(71.9 \%)$ & 0.005 \\
\hline \multicolumn{7}{|l|}{ Biochemical markers } \\
\hline Hs-cTnT (ng/L) & $30.9 \pm 40.1$ & $8.8 \pm 3.5$ & $9.4 \pm 3.0$ & $47.8 \pm 43.1$ & $55.5 \pm 53.9$ & $<0.001$ \\
\hline LDL-C (mmol/L) & $2.5 \pm 0.8$ & $2.6 \pm 0.8$ & $2.3 \pm 0.7$ & $2.4 \pm 0.8$ & $2.4 \pm 0.8$ & 0.358 \\
\hline $\mathrm{HDL}-\mathrm{C}(\mathrm{mmol} / \mathrm{L})$ & $1.3 \pm 0.4$ & $1.2 \pm 0.3$ & $1.2 \pm 0.4$ & $1.4 \pm 0.5$ & $1.3 \pm 0.5$ & 0.186 \\
\hline
\end{tabular}

Values are mean \pm SD or $\mathrm{n}(\%)$

NCA normal coronary artery, hs-cTnT high-sensitivity cardiac troponin T, CAD coronary artery disease, HCM hypertrophic cardiomyopathy, SCD sudden cardiac death, ICD implantable cardioverter defibrillator, LVEDD left ventricular end-diastolic dimension, $L A$ left atrial, MWT maximal left ventricular wall thickness, $L V E F$ left ventricular ejection fraction, $L V O T$ left ventricular outflow tract, $L D L-C$ low density lipoprotein cholesterin, $H D L-C$ high density lipoprotein cholesterin

with caution because the small events-per-variable ratio meant that the effect of overfitting might be pronounced. As a sensitivity analysis, the Lasso-Cox regression model included five variables: sex, gender, devices, beta-blocker use, and LA diameter. The results showed that the CAD and elevated hs-cTnT group still tended to be positively associated with new-onset MACEs. With the NCA and normal hs-cTnT group serving as the reference, the adjusted HR was 2.8 (95\% CI $1.0-10.2 ; P=0.048)$ for the CAD and elevated hs-cTnT group. In addition, the data showed that combined CAD could predict MACEs; thus, the net reclassification index with hs-cTnT was further evaluated. The patients have been followed up as long as 8.8 years, according to statistics from the previous study 
Table 2 Major adverse cardiovascular events

\begin{tabular}{|c|c|c|c|c|}
\hline & $\begin{array}{l}\text { NCA, normal hs-cTnT } \\
(n=54)\end{array}$ & $\begin{array}{l}\text { CAD, normal hs-cTnT } \\
(\mathrm{n}=23)\end{array}$ & $\begin{array}{l}\text { NCA, elevated hs-cTnT } \\
(n=53)\end{array}$ & $\begin{array}{l}\text { CAD, elevated } \\
\text { hs-cTnT }(n=32)\end{array}$ \\
\hline Any MACEs & 5 & 2 & 7 & 10 \\
\hline Cardiovascular death & 0 & 0 & 0 & 0 \\
\hline SCD & 0 & 0 & 1 & 1 \\
\hline Shock & 0 & 0 & 0 & 0 \\
\hline Congestive heart failure & 0 & 0 & 2 & 3 \\
\hline $\mathrm{Ml}$ & 0 & 0 & 1 & 1 \\
\hline Cardiac perioperative death & 0 & 1 & 0 & 0 \\
\hline Thrombo-embolic event & 0 & 0 & 0 & 0 \\
\hline Stroke & 4 & 1 & 2 & 3 \\
\hline TIA & 0 & 0 & 0 & 0 \\
\hline Peripheral embolism & 0 & 0 & 1 & 0 \\
\hline $\mathrm{Ml} / \mathrm{PCl}$ & 1 & 0 & 0 & 2 \\
\hline
\end{tabular}

Values are $\mathrm{n}$

MACEs major adverse cardiovascular events, MI Myocardial infarction, TIA transient ischemic attack, $P C /$ percutaneous coronary intervention; other abbreviations as in Table 1

[8], which is also confirmed in our research, showed that the 8-year accumulated incidence of events in patients who have HCM without coronary heart disease is about $20 \%$. With coronary heart disease, however, the incidence of events is $35 \%$.Therefore, the tangents of 0.2 and 0.4 were selected as low, medium, and high risk. The results of the analysis indicated that the addition of hs-cTnT increased the degree of prediction by $15.3 \%$.

\section{Additional analysis}

Patients with severe CAD were recognized to have a poor prognosis, and some of them agreed to undergo PCI at the baseline evaluation. Therefore, we excluded the patients with severe CAD $(n=10)$, and the remaining 152 patients were included in the subgroup analysis to determine the usefulness of combined evaluation in patients without severe CAD.

The subgroup analysis indicated that 23 MACEs (15.1\%) had occurred during a follow-up period of 601.2 PYs (median 3.7 years; interquartile range $2.5-5.7$ years). MACEs occurred in $5(9.3 \%, 5 / 54)$ patients in the NCA and normal hs-cTnT group, $2(8.7 \%, 2 / 23)$ patients in the CAD and normal hs-cTnT group, $7(13.2 \%, 7 / 53)$ patients in the NCA and elevated hs-cTnT group, and 9 (40.9\%, 9/22) patients in the CAD and elevated hs-cTnT group. The incidence was 13.9 (95\% CI 5.5-22.2) per 100 PYs in the CAD and elevated hs-cTnT group, which was much higher than that in the other three groups (range 2.0-3.5 per $100 \mathrm{PYs}$ ), and the clinical course was significantly worse in the CAD and elevated hs-cTnT group (log-rank $P<0.001)$. With the NCA and normal hs-cTnT group as a reference, the multivariate analysis suggested that the adjusted HR was 10.7 (95\% CI 1.7-66.8; $P=0.011)$ for the $\mathrm{CAD}$ and elevated hs-cTnT group.

Decompensated heart failure (NYHA class III/IV) may increase the release of troponin and is associated with an increased risk of death. Therefore, we conducted a sensitivity analysis restricted to patients without symptoms/ signs of heart failure. Fifty-two patients had NYHA class III/IV heart failure; after excluding these patients, 110 patients were involved in the sensitivity analysis, and 15 of these patients developed MACEs. Control with the normal group, the univariate Cox analysis suggested that for patients with abnormal troponin and coronary heart disease, the HR was 5.0 (95\% CI 1.4-17.7; $P=0.013$ ). However, no further multivariate analysis was performed because of the low number of events.

\section{Discussion}

We assessed the usefulness of combined evaluation of both CAD and hs-cTnT for prediction of MACEs in patients with HCM. We found that combined evaluation of these two markers is a more reliable predictor of MACEs than evaluation of a single marker. Thus, our findings might be of value to classify the prognosis and guide risk management in patients with HCM.

Many studies have shown that patients with HCM have a relatively benign prognosis and a mortality rate similar to that in the general population [3-5]; however, other comorbidities might pose a greater threat to survival than long-standing HCM itself $[6,7,9]$. As an important comorbidity, $\mathrm{CAD}$ can predict future adverse outcomes in patients with HCM. Sorajja et al. [8] showed that patients with concomitant severe CAD had 

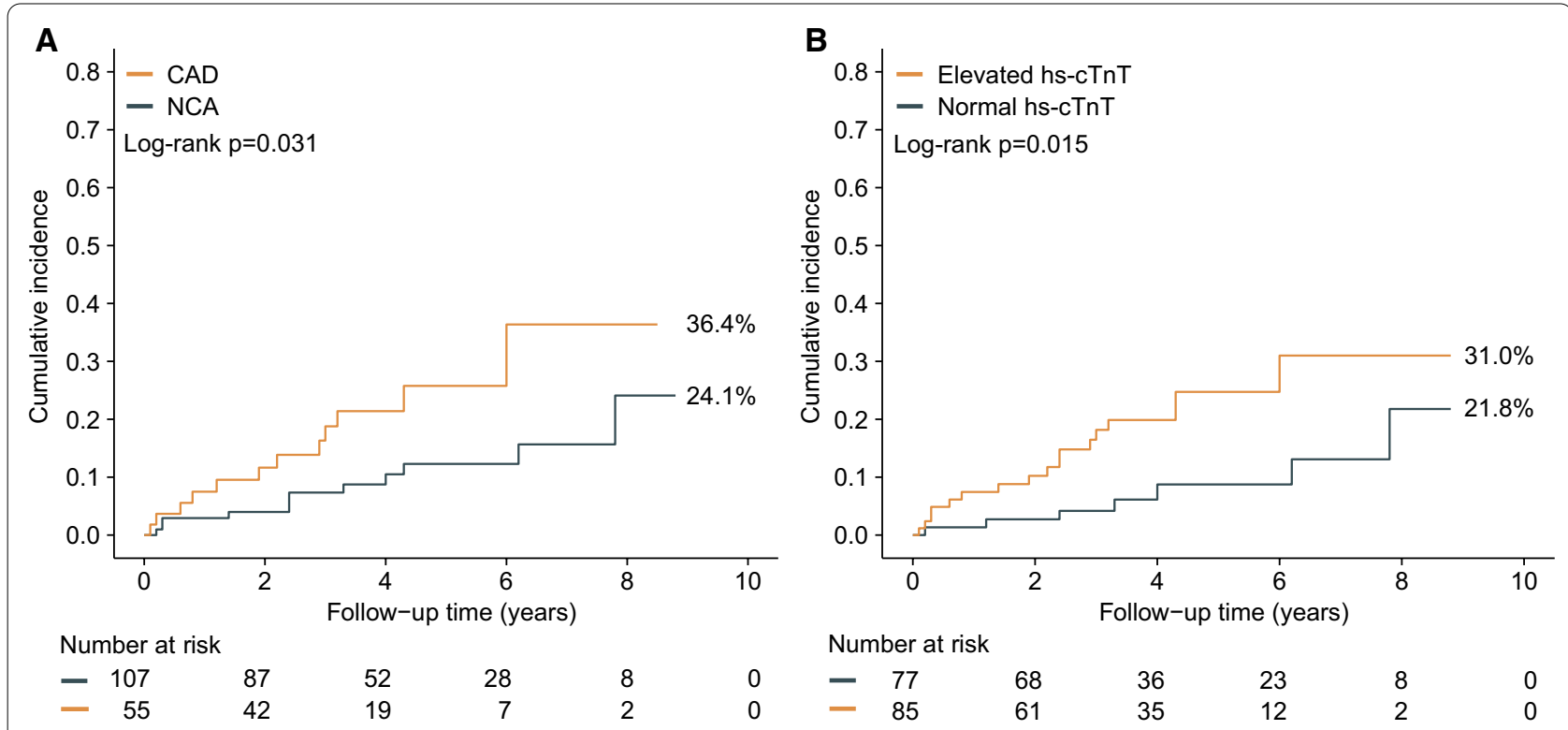

$\begin{array}{rrrrrr}-107 & 87 & 52 & 28 & 8 & 0 \\ -\quad 55 & 42 & 19 & 7 & 2 & 0\end{array}$

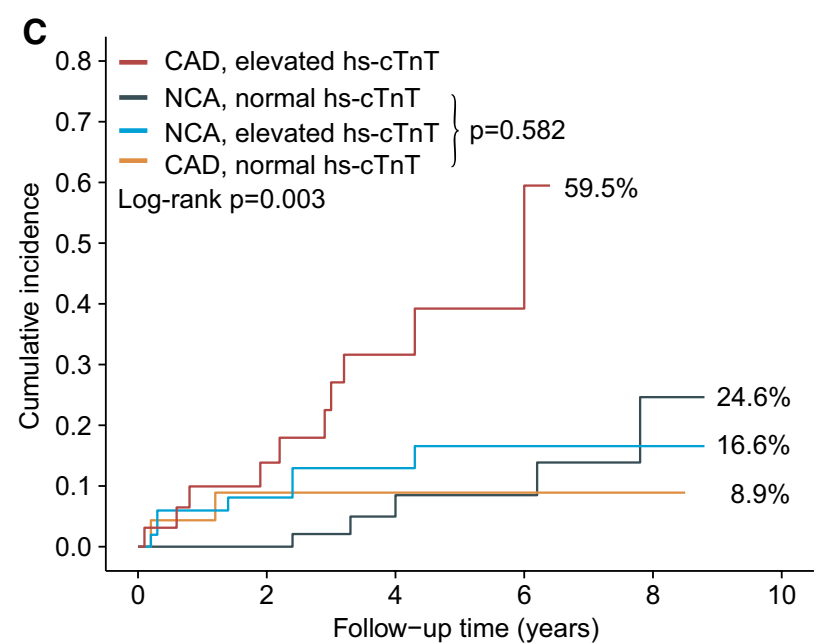

\begin{tabular}{|c|c|c|c|c|c|c|}
\hline \multicolumn{7}{|c|}{ Number at risk } \\
\hline- & 54 & 48 & 27 & 19 & 6 & 0 \\
\hline- & 23 & 20 & 9 & 4 & 2 & 0 \\
\hline- & 53 & 39 & 25 & 9 & 2 & 0 \\
\hline- & 32 & 22 & 10 & 3 & 0 & 0 \\
\hline
\end{tabular}

Fig. 2 Cumulative incidence of major adverse cardiovascular events. A Cumulative incidence of MACEs of patients with CAD versus patients with NCA. B Cumulative incidence of MACEs of patients with elevated hs-CTnT versus patients with normal hs-cTnT. C Cumulative incidence of MACEs in the four groups according to CAD and hs-cTnT. Abbreviations as in Table 1 and 2

an increased risk of death. In a recent study, Shin et al. [9] reported that severe CAD served as an independent predictor of adverse cardiovascular events in patients with HCM, and the incidence of clear CAD-related mortality or morbidity was about $25 \%$ among the events. In our study, the clinical course was significantly worse in the CAD group, and the incidence of clear CAD-related MACEs was about $20.8 \%$ among the events. Therefore, the evaluation of CAD may be important in patients with
HCM. Moreover, elevated troponin is commonly seen in patients with HCM, and an elevated hs-cTnT above a cut-off point of $14 \mathrm{ng} / \mathrm{l}$ is seen in $26 \%$ to $54 \%$ of patients with HCM [11]. The hs-cTnT concentration has been positively related to the LV wall thickness [10, 16-18], hemodynamic parameters $[10,16,17]$, clinical symptoms $[1,10,11,19]$, and outcomes $[1,10,11,19]$ in patients with HCM. Furthermore, the risk of adverse cardiovascular events seems to be greater with increased degrees of 
Table 3 Associations of both coronary artery disease and high-sensitivity cardiac troponin T with major adverse cardiovascular events

\begin{tabular}{|c|c|c|c|c|}
\hline & $\begin{array}{l}\text { NCA, normal } \\
\text { hs-cTnT }(n=54)\end{array}$ & CAD, normal hs-cTnT $(n=23)$ & $\begin{array}{l}\text { NCA, elevated } \\
\text { hs-cTnT }(n=53)\end{array}$ & CAD, elevated hs-cTnT $(n=32)$ \\
\hline MACEs (n) & 5 & 2 & 7 & 10 \\
\hline Person-years & 246.8 & 90.4 & 199.3 & 95.8 \\
\hline Incidence rate $(95 \% \mathrm{Cl})^{\mathrm{a}}$ & $2.0(0.3-3.8)$ & $2.2(0.0-5.2)$ & $3.5(1.0-6.1)$ & $10.4(4.3-16.6)$ \\
\hline Unadjusted HR (95\% CI), P & 1 & $1.1(0.2-5.7), 0.908$ & $1.9(0.6-6.0), 0.287$ & $5.5(1.8-16.8), 0.003$ \\
\hline \multicolumn{5}{|l|}{ Adjusted HR $(95 \%$ Cl), P } \\
\hline Model 1 & 1 & $0.6(0.1-3.3), 0.526$ & $1.6(0.5-5.2), 0.436$ & $3.4(1.0-11.1), 0.044$ \\
\hline Model 2 & 1 & $0.5(0.1-3.4), 0.521$ & $1.6(0.5-5.1), 0.461$ & $3.7(1.0-13.1), 0.046$ \\
\hline Model 3 & 1 & $0.6(0.1-4.6), 0.664$ & $1.9(0.5-7.1), 0.322$ & $5.7(1.3-24.1), 0.018$ \\
\hline Model 4 & 1 & $0.5(0.1-3.9), 0.542$ & $1.2(0.3-5.2), 0.765$ & $\begin{array}{l}\text { Model } 1 \text { with adjustment for age } \\
\text { and gender5.0 (1.0-23.8), } 0.046\end{array}$ \\
\hline
\end{tabular}

Model 1 with adjustment for age and gender

Model 2 with adjustment for model 1 plus symptoms and comorbidities, including chest pain, palpitations, syncope/pre-syncope, dyspnea, hypertension, diabetes and atrial fibrillation

Model 3 with adjustment for model 2 plus devices, procedures and medications, including aspirin, warfarin, statins and beta-blocker

Model 4 with adjustment for model 3 plus echocardiographic parameters, including LVEDD, LA diameter, MWT, LVEF and LVOT obstruction

$\mathrm{Cl}$ confidence interval, $H R$ hazard ratio; other abbreviations as in Tables 1 and 2

a Per 100 person-years

abnormality in the hs-cTnT concentration. Our study also showed similar results; therefore, hs-cTnT is expected to be useful in the clinical evaluation of patients with HCM. Although our multivariate analysis showed that statistical significance was not achieved, our findings suggested that both CAD and elevated hs-cTnT tended to be positively associated with MACEs. We believe that this might have been caused by the relatively small sample and that our results could be improved by a larger sample. Notably, however, the combined evaluation of the two markers significantly improved the predictive values. This combined evaluation achieved statistical significance in the same sample and therefore indicates the clinical usefulness of combined evaluation.

In the present study, combined evaluation of both CAD and hs-cTnT had better predictive value than evaluation of a single marker. When patients with HCM have CAD, the coronary blood flow might decrease to different degrees, which could cause MACEs. Furthermore, patients with HCM who develop CAD usually have more cardiovascular risk factors $[8,20]$. From this viewpoint, our study supports previous findings. These cardiovascular risk factors can exacerbate the structural abnormalities and inherent endothelial dysfunction in patients with $\operatorname{HCM}[8,21]$, further increasing the detrimental impact on the prognosis. However, the mechanisms underlying the release of hs-cTnT in patients with HCM remain unclear. It is speculated that the increase may be caused by relative myocardial ischemia resulting from an imbalance between inappropriate hypertrophy of the myocardium and insufficient coronary arterial supply $[10,12]$.
In this respect, the present study proposes microvascular dysfunction as an important explanation [12]. Microvascular dysfunction associated with elevated hs-cTnT can result in myocyte injury and subsequent fibrosis in $\mathrm{HCM}$, leading to poor outcomes [10,12]. Given the increased myocardial mass and high myocardial oxygen demand, patients with microvascular dysfunction might be particularly susceptible to the additional ischemic burden of CAD [22], further increasing the incidence of adverse outcomes. As mentioned above, epicardial CAD might respect a diminished coronary flow and a cluster of cardiovascular risk factors, whereas elevated hs-cTnT could respect myocardial injury and microvascular dysfunction; these might represent the total ischemic burden of the myocardium. Therefore, combined evaluation of both $\mathrm{CAD}$ and hs-cTnT should have greater prediction ability than evaluation of a single marker. Notably, performing coronary artery imaging in patients with $\mathrm{HCM}$ who have both elevated hs-cTnT and risk factors for CAD is taken for granted. Therefore, we should pay more attention to patients with either elevated hs-cTnT or risk factors for CAD, especially asymptomatic patients; such patients may need further coronary artery imaging.

This study has several limitations. First, this was a single-center retrospective clinical study and therefore may have certain inherent biases. We could only use the patients' existing data. In total, 321 patients were excluded because of a lack of coronary artery imaging and hs-cTnT data, which may represent a selection bias. Although this was a specially selected group of patients, it still indicates the risk of CAD combination for this 
type of patient. Additionally, some patients' baseline data excluded renal function parameters. However, renal insufficiency was not diagnosed in the medical records; therefore, we can partially rule out renal insufficiency as a cause of the rise in troponin. These limitations warrant prospective studies. Second, coronary imaging and hscTnT measurement should ideally have been performed during a steady state. In our study, 52 (32.1\%) patients had NYHA class III/IV heart failure, which may suggest the acute phase of disease. Therefore, larger prospective studies are needed to confirm and extend the present findings. Third, the number of MACEs was so small $(n=24)$ that the subanalysis and grouping analysis may have limited the statistical value; however, these analyses still provided useful findings. Although the multivariate analysis showed that statistical significance was not achieved, our findings suggest that both CAD and elevated hs-cTnT tended to be positively associated with MACEs. We believe that this could be improved by a larger sample. Because of the small number of events, the role of combined evaluation of CAD and hs-cTnT in specific subgroups of patients with HCM remains to be further evaluated. Fourth, not all patients agreed to undergo coronary angiography, and 33 (20.4\%) patients underwent coronary $\mathrm{CT}$ angiography; this might suggest that the study population was somewhat heterogeneous. However, coronary CT angiography has high negative and positive predictive values in patients with HCM [22], and the use of coronary CT angiography should be acceptable. Fifth, because the severity of CAD was assessed by coronary angiography or coronary $\mathrm{CT}$ angiography without intravascular ultrasound or optical coherence tomography, the specific causes of stenosis cannot be determined. However, the performance of intravascular ultrasound and optical coherence tomography for all patients was not feasible for pragmatic and logistical reasons. Finally, severe CAD usually requires intervention and has a very poor prognosis. Therefore, the combination of patients with severe CAD and mild to moderate $C A D$ into a single group may have caused bias. However, the additional analysis indicated similar results, further supporting the main analysis.

\section{Conclusions}

Combined evaluation of both CAD and hs-cTnT could be a more reliable predictor of MACEs than evaluation of a single marker in patients with HCM. The present study findings may be of value to classify the prognosis and guide risk management in patients with HCM. Larger studies are needed to confirm these findings.

\section{Abbreviations}

CAD: Coronary artery disease; hs-cTnT: High-sensitivity cardiac troponin T; MACEs: Major adverse cardiovascular events; HCM: Hypertrophic cardiomyopathy; PYs: Person-years; NCA: Normal coronary artery; LV: Left ventricular; LVEDD: LV end-diastolic dimension; LVEF: LV ejection fraction; LVOT: LV outflow tract; LA: Left atrial; MWT: Maximum LV wall thickness; PCI: Percutaneous coronary intervention; SCD: Sudden cardiac death; ICD: Implantable cardioverterdefibrillator.

\section{Acknowledgements \\ We thank Angela Morben, DVM, ELS, from Liwen Bianji, Edanz Editing China (www.liwenbianji.cn/ac), for editing the English text of a draft of this manuscript.}

\section{Authors' contributions}

HL: Concept/design, Data analysis/interpretation, Data collection,Drafting article. HCT: Concept/design.ZW: Concept/ design, Data collection, Drafting article. XC: Data analysis/interpretation, Critical revision of article. YH: Concept/ design, Data analysis/interpretation. SH: Concept/design, Data analysis/ interpretation, Approval of article. All authors read and approved the final manuscript.

\section{Funding}

This work was supported by the National Natural Science Foundation of China (Grant No. 81600299).

\section{Availability of data and materials}

The datasets used and/or analysed during the current study are available from the corresponding author on reasonable request.

\section{Declarations}

\section{Ethics approval and consent to participate}

The study was approved by the Ethics Committee on Medical Research of West China Hospital of Sichuan University and conformed to the principles of the Helsinki declaration. The requirement for informed consent was waived because of the retrospective nature of the study.

\section{Consent for publication}

Not applicable.

\section{Competing interests}

The authors declare that they have no competing interests.

\section{Author details}

${ }^{1}$ Department of Cardiology, West China Hospital of Sichuan University, Chengdu 610041, China. ${ }^{2}$ Department of Cardiology, National University Heart Centre, Singapore, Singapore.

Received: 17 December 2019 Accepted: 24 June 2021

Published online: 03 July 2021

\section{References}

1. Authors/Task Force m, Elliott PM, Anastasakis A, Borger MA, Borggrefe M, Cecchi F, Charron P, Hagege AA, Lafont A, Limongelli G, Mahrholdt $\mathrm{H}$, McKenna WJ, Mogensen J, Nihoyannopoulos P, Nistri S, Pieper PG, Pieske B, Rapezzi C, Rutten FH, Tillmanns C, Watkins H. 2014 ESC Guidelines on diagnosis and management of hypertrophic cardiomyopathy: the Task Force for the Diagnosis and Management of Hypertrophic Cardiomyopathy of the European Society of Cardiology (ESC). Eur Heart J. 2014;35:2733-79.

2. Semsarian C, Ingles J, Maron MS, Maron BJ. New perspectives on the prevalence of hypertrophic cardiomyopathy. J Am Coll Cardiol. 2015;65:1249-54.

3. Maron BJ. Clinical course and management of hypertrophic cardiomyopathy. N Engl J Med. 2018;379:655-68.

4. Spirito P. The dawn of a better day for patients with hypertrophic cardiomyopathy. J Am Coll Cardiol. 2015;65:1929-30. 
5. Maron BJ, Maron MS, Rowin EJ. Perspectives on the overall risks of living with hypertrophic cardiomyopathy. Circulation. 2017;135:2317-9.

6. Ball W, Ivanov J, Rakowski H, Wigle ED, Linghorne M, Ralph-Edwards A, Williams WG, Schwartz L, Guttman A, Woo A. Long-term survival in patients with resting obstructive hypertrophic cardiomyopathy comparison of conservative versus invasive treatment. J Am Coll Cardiol. 2011;58:2313-21.

7. Maron BJ, Rowin EJ, Casey SA, Haas TS, Chan RH, Udelson JE, Garberich RF, Lesser JR, Appelbaum E, Manning WJ, Maron MS. Risk stratification and outcome of patients with hypertrophic cardiomyopathy $>=60$ years of age. Circulation. 2013;127:585-93.

8. Sorajja P, Ommen SR, Nishimura RA, Gersh BJ, Berger PB, Tajik AJ. Adverse prognosis of patients with hypertrophic cardiomyopathy who have epicardial coronary artery disease. Circulation. 2003;108:2342-8.

9. Shin YJ, Lee JH, Yoo JY, Kim JA, Jeon Y, Yoon YE, Chun EJ. Clinical significance of evaluating coronary atherosclerosis in adult patients with hypertrophic cardiomyopathy who have chest pain. Eur Radiol. 2019;29:4593-602.

10. Kubo T, Kitaoka H, Yamanaka S, Hirota T, Baba Y, Hayashi K, liyama T, Kumagai N, Tanioka K, Yamasaki N, Matsumura Y, Furuno T, Sugiura T, Doi YL. Significance of high-sensitivity cardiac troponin T in hypertrophic cardiomyopathy. J Am Coll Cardiol. 2013;62:1252-9.

11. Kehl DW, Buttan A, Siegel RJ, Rader F. Clinical utility of natriuretic peptides and troponins in hypertrophic cardiomyopathy. Int J Cardiol. 2016;218:252-8.

12. Tardiff JC, Carrier L, Bers DM, Poggesi C, Ferrantini C, Coppini R, Maier LS, Ashrafian $\mathrm{H}$, Huke $\mathrm{S}$, van der Velden J. Targets for therapy in sarcomeric cardiomyopathies. Cardiovasc Res. 2015;105:457-70.

13. He $S$, Wang $Z$, Cheem $T H$, Liao H, Chen $X$, He Y. External validation of the model of thrombo-embolic risk in hypertrophic cardiomyopathy patients. Can J Cardiol. 2019;5:5. https://doi.org/10.1016/j.cjca.2019.05. 035 ((in press)).

14. Saenger AK, Beyrau R, Braun S, Cooray R, Dolci A, Freidank H, Giannitsis E, Gustafson S, Handy B, Katus H, Melanson SE, Panteghini M, Venge P, Zorn M, Jarolim P, Bruton D, Jarausch J, Jaffe AS. Multicenter analytical evaluation of a high-sensitivity troponin T assay. Clin Chim Acta. 2011;412:748-54
15. Pavlou M, Ambler G, Seaman SR, Guttmann O, Elliott P, King M, Omar RZ How to develop a more accurate risk prediction model when there are few events. BMJ. 2015;351:h3868.

16. Jenab Y, Pourjafari M, Darabi F, Boroumand MA, Zoroufian A, Jalali A. Prevalence and determinants of elevated high-sensitivity cardiac troponin T in hypertrophic cardiomyopathy. J Cardiol. 2014;63:140-4.

17. Zhang C, Liu R, Yuan J, Cui J, Hu F, Yang W, Zhang Y, Yang C, Qiao S. Significance and determinants of cardiac Troponin I in patients with obstructive hypertrophic cardiomyopathy. Am J Cardiol. 2015;116:1744-51.

18. Hladij R, Rajtar-Salwa R, Dimitrow PP. Troponin as ischemic biomarker is related with all three echocardiographic risk factors for sudden death in hypertrophic cardiomyopathy (ESC Guidelines 2014). Cardiovasc Ultrasound. 2017:15:24.

19. Kubo T, Kitaoka H, Okawa M, Yamanaka S, Hirota T, Baba Y, Hayato K, Yamasaki N, Matsumura Y, Yasuda N, Sugiura T, Doi YL. Combined measurements of cardiac troponin I and brain natriuretic peptide are useful for predicting adverse outcomes in hypertrophic cardiomyopathy. Circ J. 2011;75:919-26.

20. Okayama S, Soeda T, Kawakami R, Takami Y, Somekawa S, Ueda T, Sugawara Y, Matsumoto T, Sung JH, Nishida T, Uemura S, Saito Y. Evaluation of coronary artery disease and cardiac morphology and function in patients with hypertrophic cardiomyopathy, using cardiac computed tomography. Heart Vessels. 2015;30:28-35.

21. Rizzoni D, Palombo C, Porteri E, Muiesan ML, Kozakova M, La Canna G, Nardi M, Guelfi D, Salvetti M, Morizzo C, Vittone F, Rosei EA. Relationships between coronary flow vasodilator capacity and small artery remodelling in hypertensive patients. J Hypertens. 2003;21:625-31.

22. Finocchiaro G, Magavern E, Sinagra G, Ashley E, Papadakis M, Tome-Esteban M, Sharma S, Olivotto I. Impact of demographic features, lifestyle, and comorbidities on the clinical expression of hypertrophic cardiomyopathy. J Am Heart Assoc. 2017;6:e007161.

\section{Publisher's Note}

Springer Nature remains neutral with regard to jurisdictional claims in published maps and institutional affiliations.
Ready to submit your research? Choose BMC and benefit from:

- fast, convenient online submission

- thorough peer review by experienced researchers in your field

- rapid publication on acceptance

- support for research data, including large and complex data types

- gold Open Access which fosters wider collaboration and increased citations

- maximum visibility for your research: over $100 \mathrm{M}$ website views per year

At BMC, research is always in progress.

Learn more biomedcentral.com/submissions 